\title{
Room-temperature multiferroic behavior in layer-structured Aurivillius phase ceramics
}

Cite as: Appl. Phys. Lett. 117, 052903 (2020); https://doi.org/10.1063/5.0017781

Submitted: 09 June 2020. Accepted: 25 July 2020 . Published Online: 07 August 2020

Zheng Li, Vladimir Koval (D), Amit Mahajan, Zhipeng Gao, Carlo Vecchini, Mark Stewart, Markys G. Cain (D), Kun Tao, Chenglong Jia (D), Giuseppe Viola, and Haixue Yan (D)
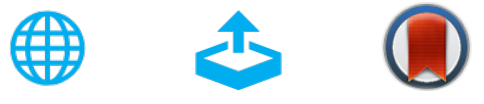

View Online

Export Citation

\section{ARTICLES YOU MAY BE INTERESTED IN}

Intrinsic piezoelectricity in $(\mathrm{K}, \mathrm{Na}) \mathrm{NbO}_{3}$-based lead-free single crystal: Piezoelectric anisotropy and its evolution with temperature

Applied Physics Letters 117, 052904 (2020); https://doi.org/10.1063/5.0012124

Current-induced bulk magnetization of a chiral crystal $\mathrm{CrNb}_{3} \mathrm{~S}_{6}$

Applied Physics Letters 117, 052408 (2020); https://doi.org/10.1063/5.0017882

Magnetic transition behavior and large topological Hall effect in hexagonal $\mathrm{Mn}_{2-x} \mathrm{Fe}_{1+x} \mathrm{Sn}(\mathrm{x}=$ 0.1) magnet

Applied Physics Letters 117, 052407 (2020); https://doi.org/10.1063/5.0011570

\section{Lock-in Amplifiers up to $600 \mathrm{MHz}$}
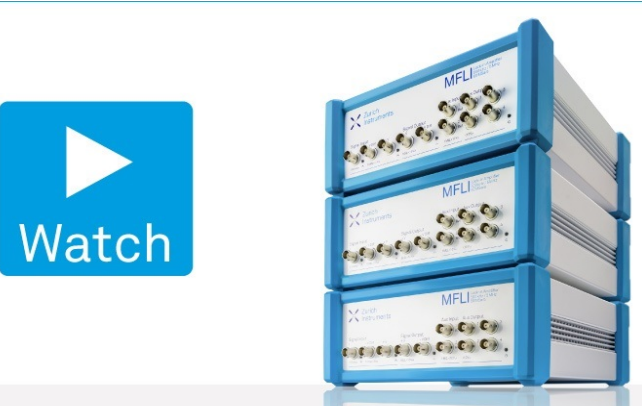


\title{
Room-temperature multiferroic behavior in layer-structured Aurivillius phase ceramics
}

Cite as: Appl. Phys. Lett. 117, 052903 (2020); doi: 10.1063/5.0017781

Submitted: 9 June 2020 . Accepted: 25 July 2020 .

Published Online: 7 August 2020 · Corrected: 11 August 2020

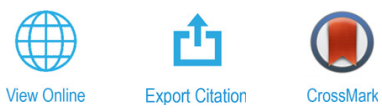

Zheng Li, Vladimir Koval, ${ }^{7}$ (D) Amit Mahajan, ${ }^{3}$ Zhipeng Gao, ${ }^{4}$ Carlo Vecchini, ${ }^{5}$ Mark Stewart, ${ }^{5}$ Markys G. Cain, ${ }^{6}$ Kun Tao, ${ }^{7}$ Chenglong Jia, ${ }^{7, a)}$ (D) Giuseppe Viola, ${ }^{3}$ and Haixue Yan ${ }^{3, b)}$ (DD

\author{
AFFILIATIONS \\ ${ }^{7}$ Gemological Institute, China University of Geosciences, Wuhan 430074, China \\ ${ }^{2}$ Institute of Materials Research, Slovak Academy of Sciences, Watsonova 47, Kosice 04001, Slovakia \\ ${ }^{3}$ School of Engineering and Materials Science, Queen Mary, University of London, London E1 4NS, United Kingdom \\ ${ }^{4}$ National Key Laboratory of Shock Wave and Detonation Physics Institute of Fluid Physics, China Academy of Engineering Physics, \\ Mianyang 621900, China \\ ${ }^{5}$ National Physical Laboratory, Hampton Road, Teddington TW11 OLW, United Kingdom \\ ${ }^{6}$ Electrosciences Ltd, Farnham, Surrey GU9 9QT, United Kingdom \\ ${ }^{7}$ School of Physical Science and Technology, Lanzhou University, Lanzhou 730000, China \\ a)Email: cljia@lzu.edu.cn \\ ${ }^{\text {b) }}$ Author to whom correspondence should be addressed: h.x.yan@qmul.ac.uk
}

\begin{abstract}
Multiferroics that simultaneously exhibit ferroelectricity and ferromagnetism have recently attracted great attention due to their potential application in next generation electronic devices. However, only a few single-phase multiferroic materials exhibit ferroelectric and ferromagnetic orders at room temperature. Recently, some bismuth layer-structured Aurivillius compounds were reported as multiferroics at room temperature, but the origin of their magnetic property is still under debate because the net magnetization may originate from the presence of secondary phases that are not easily detected by laboratory XRD diffractometers. Here, textured Aurivillius phase $\mathrm{Bi}_{5.25} \mathrm{La}_{0.75} \mathrm{FeCoTi}_{3} \mathrm{O}_{18}$ ceramics were prepared by Spark Plasma Sintering. The ferromagnetic character of the ceramics was indicated by the magnetic field-induced reversible intensity changes of a certain set of crystalline planes belonging to the Aurivillius phase, as measured by in situ neutron diffraction under the applied magnetic field. The first principles calculations indicate that the ferromagnetism originates from double exchange interactions $\mathrm{Fe}^{3+}-\mathrm{O}-\mathrm{Fe}^{3+}, \mathrm{Co}^{3+}-\mathrm{O}-\mathrm{Co}^{3+}$, and $\mathrm{Fe}^{3+}-\mathrm{O}-\mathrm{Co}^{3+}$ in the ferro-toroidal main phase. The magnetic-controlled ferroelectric domain switching was observed by piezoelectric force microscopy at room temperature. The prepared Aurivillius phase ceramics, with Co/Fe contributing to magnetization and polarization at the same time, can be considered an intrinsic room-temperature multiferroic.
\end{abstract}

Published under license by AIP Publishing. https://doi.org/10.1063/5.0017781

Multiferroics that simultaneously exhibit ferroelectricity (FE) and ferromagnetism (FM) have recently attracted great attention due to their potential application in new types of sensors, actuators, memory storage devices, etc. ${ }^{1-4}$ However, only a few single-phase multiferroic materials exhibit ferroelectric and ferromagnetic orders at room temperature. ${ }^{5}$ Recently, bismuth layer-structured Aurivillius compounds of a general formula $\left(\mathrm{Bi}_{2} \mathrm{O}_{2}\right)^{2+}\left(\mathrm{A}_{\mathrm{m}-1} \mathrm{~B}_{\mathrm{m}} \mathrm{O}_{3 \mathrm{~m}+1}\right)^{2-}$ (where $\mathrm{A}$ is a mono-, di-, or trivalent cation with 12 -fold-coordination, $\mathrm{B}$ is a transition-metal cation with sixfold-coordination, and $\mathrm{m}$ is the number of octahedral layers in the perovskite slab) ${ }^{6}$ with the addition of magnetic species on the B-site have been investigated and shown to be one of the new leading alternatives to $\mathrm{BiFeO}_{3}$ as a single-phase multiferroic material. ${ }^{7-11}$
The Aurivillius phase materials $\mathrm{Bi}_{5} \mathrm{FeTi}_{3} \mathrm{O}_{15}(\mathrm{~m}=4)$ and $\mathrm{Bi}_{6} \mathrm{Fe}_{2} \mathrm{Ti}_{3} \mathrm{O}_{18}$ $(\mathrm{m}=5)$, which can be considered as a solid solution of $\mathrm{Bi}_{4} \mathrm{Ti}_{3} \mathrm{O}_{12}$ and $\mathrm{BiFeO}_{3}$, showed the coexistence of FE and FM orders. ${ }^{12,13}$ By substituting $\mathrm{Co}$ and $\mathrm{Fe}$ in the $\mathrm{B}$-site, the ferromagnetism of $\mathrm{Bi}_{5} \mathrm{Fe}_{0.5} \mathrm{Co}_{0.5} \mathrm{Ti}_{3} \mathrm{O}_{15}$ $(\mathrm{m}=4)$ and $\mathrm{Bi}_{6} \mathrm{FeCoTi}_{3} \mathrm{O}_{18}(\mathrm{~m}=5)$ was remarkably improved with both compositions showing multiferroic properties at room temperature. ${ }^{14,15}$ However, the origin of their magnetic property is still under debate because the net magnetization may originate from the presence of secondary phases that are not easily detected by laboratory XRD diffractometers. ${ }^{16}$ Therefore, more research was needed to further understand the origins of the multiferroic properties of Aurivillius phase materials. 
In this study, textured Aurivillius phase $\mathrm{Bi}_{5.25} \mathrm{La}_{0.75} \mathrm{FeCoTi}_{3} \mathrm{O}_{18}$ (BLFCT) ceramics were prepared by Spark Plasma Sintering (SPS). Partial La substitution was chosen to reduce the electrical conductivity and to decrease the ferroelectric coercive field, while Co, in cosubstitution with $\mathrm{Fe}$, was selected to increase magnetization. ${ }^{14,17}$ Due to the structural anisotropy of Aurivillius phase materials and the presence of ferroelectric polarization in the $a-b$ plane, the SPS textured BLFCT ceramic would allow easier switching of the polarization in the $a-b$ plane if compared to that prepared by conventional sintering. The ferroelectric behavior of the Aurivillius phase is confirmed by the presence of current peaks in the current vs electric-field loops, which indicate the occurrence of ferroelectric domain switching. ${ }^{18}$ The ferromagnetic character was indicated by the magnetic field-induced reversible intensity changes of a certain set of crystalline planes belonging to the Aurivillius phase, measured by in situ neutron diffraction under the applied magnetic field on the WISH instrument at the ISIS Neutron Spallation facility, RAL, Didcot, Oxfordshire, UK. Ferroelectric domain switching induced by an applied external magnetic field suggests the existence of spontaneous toroidal moments and a linear magnetoelectric coupling in the material at room temperature (RT). Therefore, we can conclude that the textured Aurivillius BLFCT phase material prepared by SPS is an intrinsic multiferroic with the coupled ferroelectric and magnetic order parameters at room temperature.

The BLFCT samples were obtained using the conventional solid state reaction route and SPS. The details of sample preparation, characterization, and first principles modeling of magnetization are shown in the supplementary material.

Figure 1 shows the $\mathrm{x}$-ray diffraction (XRD) pattern of the BLFCT powder. All the peaks were indexed to an orthorhombic structure with a $B 2 c b$ space group characteristic of five-layered Aurivillius phase compounds, and simultaneously to another orthorhombic phase with a crystallographic symmetry of an $A 2_{1}$ am space group, typical of fourlayered Aurivillius oxides. ${ }^{1,20}$ The lattice parameters of the orthorhombic $B 2 c b$ structures $a=5.4530(2) \AA, \quad b=5.4427(1) \AA$, and $c=50.670(2) \AA$ and the four-layered $A 2_{1} a m$ phases $a=5.4651(6) \AA$, $b=5.3943(6) \AA$, and $c=41.487(2) \AA$ were obtained by the full pattern matching method using the FullProf software package (http:// www.ill.eu).

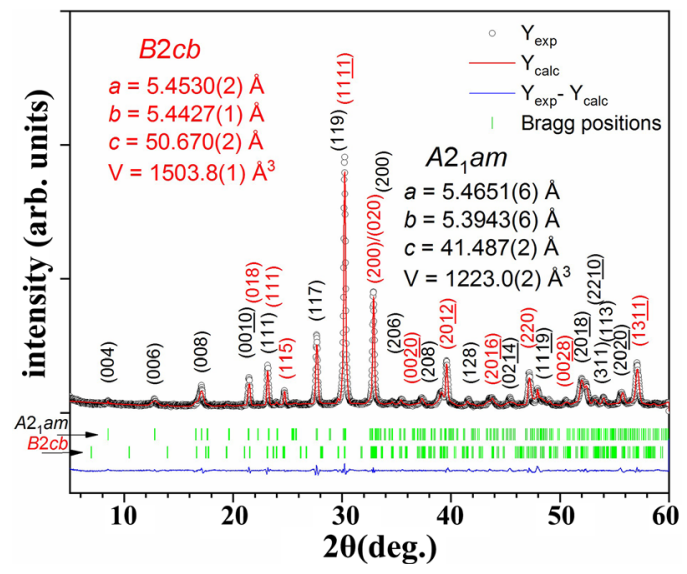

FIG. 1. X-ray diffraction pattern of the BLFCT powder.
These results indicate that BLFCT is a mixed-layer Aurivillius material consisting of the $\mathrm{m}=4$ and $\mathrm{m}=5$ perovskite slabs. No extra peaks of the possible impurity phases were identified within the XRD's instrumental resolution. Figure S1 shows the SEM images of the textured BLFCT ceramics. The plate-like grains $(a-b$ axes) are aligned in the direction perpendicular to the SPS pressure direction. Moreover, a small amount of a secondary phase also marked by the red circle in Fig. S1 can be observed in the microstructure. The amount of this secondary phase is estimated to be approximately $1.4 \mathrm{vol}$. \%, which is below the trace level of XRD. EDX line scanning analysis (Fig. S2 and Table S1) revealed that local chemical inhomogeneities are composed of $\mathrm{Fe}, \mathrm{Co}, \mathrm{O}$, and Ti. Thus, the secondary phase is assumed to be of the $\mathrm{Co}_{2} \mathrm{FeO}_{4}$ spinel ferrite type with cobalt ions partially replaced by titanium ions. Such a chemical impurity has also been reported to exist in a four-layered Aurivillius compound $\mathrm{Bi}_{5} \mathrm{Fe}_{0.5} \mathrm{Co}_{0.5} \mathrm{Ti}_{3} \mathrm{O}_{15}{ }^{16}$

Figure 2(a) shows the temperature dependence of the dielectric permittivity and loss of BLFCT ceramics measured perpendicular to the SPS pressure direction at five different frequencies (50, 70100 , 300 , and $500 \mathrm{kHz}$ ). The peak of the dielectric permittivity at about $1060 \mathrm{~K}$ corresponds to the FE $T_{\mathrm{c}}$ of BLFCT. Hence, the ferroelectric transition temperature of BLFCT is higher than that of $\mathrm{Bi}_{6} \mathrm{Ti}_{3} \mathrm{Fe}_{2} \mathrm{O}_{18}$ phase (at $973 \mathrm{~K}) .^{13}$ Figure 2(b) shows the $P-E$ and $I-E$ hysteresis loops of the textured BLFCT ceramics measured in a perpendicular direction to the SPS pressure at RT. The observed current peaks in the $I-E$ loops confirm the ferroelectric domain switching, ${ }^{21,22}$ which occurs in the sample upon field reversal. The maximum value of the polarization of BLFCT is $10 \mu \mathrm{C} / \mathrm{cm}^{2}$.

Figure 2(c) shows the temperature dependence of the zero-field cooled (ZFC) and then field-cooled (FC) magnetizations under an applied magnetic field of 200 Oe for BLFCT. We propose that BLFCT
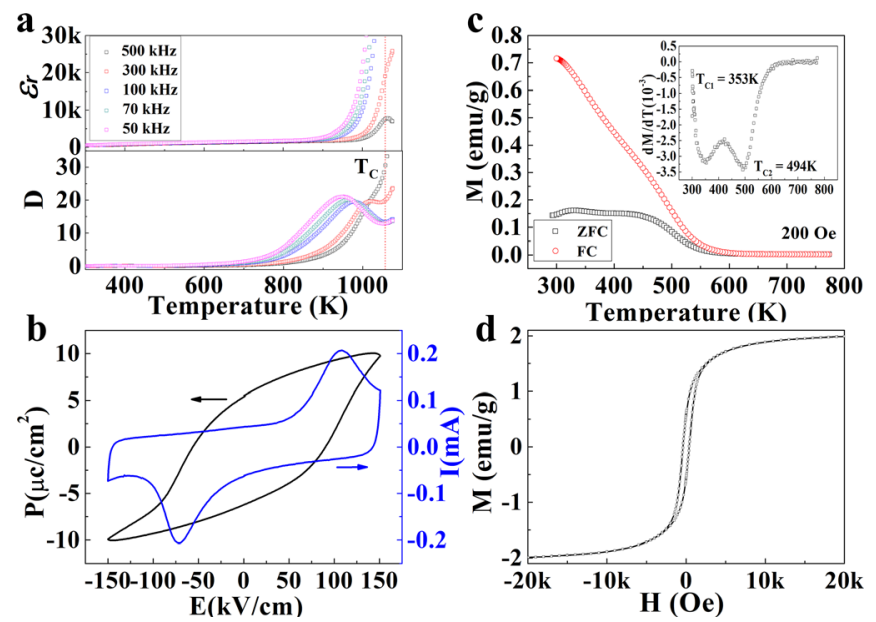

FIG. 2. (a) The temperature dependence of the relative dielectric permittivity (above) and loss tangent (down) of BLFCT ceramics measured perpendicular $[\perp]$ to the SPS pressure direction. (b) The P-E and I-E hysteresis loops of the textured BLFCT ceramics measured perpendicular to the SPS pressure direction. The curves demonstrate the domain switching characteristic of a ferroelectric. (c) The temperature dependence of the magnetization for BLFCT ceramics $[\perp]$ in the ZFC/ $\mathrm{FC}$ modes measured in $\mathrm{H}=200 \mathrm{Oe}$. The inset shows the first derivative of magnetization $\mathrm{dM} / \mathrm{dT}$ as a function of temperature. (d) The field dependence of magnetization at $300 \mathrm{~K}$ for the BLFCT sample. 
undergoes a paramagnetic to ferromagnetic transition at $\mathrm{T}_{\mathrm{cl}} \sim 494 \mathrm{~K}$ (defined as the temperature corresponding to the peak of $\mathrm{dM} / \mathrm{dT}$ ), which is close to that of $\mathrm{Bi}_{6} \mathrm{FeCoTi}_{3} \mathrm{O}_{18}(526 \mathrm{~K}){ }^{23}$ The ferromagnetism of BLFCT is induced by the double exchange interactions between neighboring $\mathrm{Fe}^{3+}-\mathrm{O}-\mathrm{Fe}^{3+}, \mathrm{Co}^{3+}-\mathrm{O}-\mathrm{Co}^{3+}$, and $\mathrm{Fe}^{3+}-\mathrm{O}-\mathrm{Co}^{3+}$ (cf. the following density functional theory calculations). ${ }^{24}$ This is consistent with the EDX result for the elemental makeup of the main phase. Another peak clearly observable on the ZFC curve at $\mathrm{T}_{\mathrm{c} 2} \sim 353 \mathrm{~K}$ can be associated with the magnetic transition of the secondary phase $\mathrm{Ti}$ doped $\mathrm{Co}_{2} \mathrm{FeO}_{4}$. The fact that $\mathrm{T}_{\mathrm{c} 2}$ is lower than the transition temperature of $\mathrm{Co}_{2} \mathrm{FeO}_{4}(460 \mathrm{~K})$ can be explained by $\mathrm{Ti}$ substitution. ${ }^{16,25}$ The spontaneous magnetization $\left(\mathrm{M}_{\mathrm{s}}\right)$ of $\mathrm{Co}_{2} \mathrm{FeO}_{4}$ is about 16-23.5 emu/g. ${ }^{25}$ Therefore, the spontaneous magnetization of $1.4 \mathrm{vol} . \%$ $\mathrm{Co}_{2-\mathrm{x}} \mathrm{FeTi}_{\mathrm{x}} \mathrm{O}_{4}$ should be about $0.22-0.32 \mathrm{emu} / \mathrm{g}$, which is much smaller than the spontaneous magnetization of BLFCT $\left[\mathrm{M}_{\mathrm{s}}=1.85 \mathrm{emu} / \mathrm{g}\right.$, Fig. 2(d) $]$. In order to distinguish the contribution of secondary phase and main phase, the $\mathrm{M}-\mathrm{H}$ loop was measured at a temperature between the two phase transitions $\mathrm{T}_{\mathrm{cl}}$ and $\mathrm{T}_{\mathrm{c} 2}$ (Fig. S3). The spontaneous magnetization of the ceramic measured at $425 \mathrm{~K}$ is about $1.58 \mathrm{emu} / \mathrm{g}$. This means that the magnetic contribution of the secondary phase is about $0.27 \mathrm{emu} / \mathrm{g}$, which agrees with the secondary phase amount obtained from the EDX results. Therefore, the main ferromagnetism of BLFCT is believed to originate from the Aurivillius phase.

Figure 3 demonstrates an example of the ferromagnetic exchange mechanism between neighboring $\mathrm{Fe}^{3+}-\mathrm{O}-\mathrm{Co}^{3+}$ by the density functional theory (DFT) calculations with the Vienna $a b$ initio simulation package (VASP) code. The effective Hubbard interaction of $\mathrm{U}_{\mathrm{Fe}}=2 \mathrm{eV}$ and $\mathrm{U}_{\mathrm{Co}}=3 \mathrm{eV}$ for $\mathrm{Fe}$ and Co atoms, respectively, was introduced to take strong correlation into account in the generalized gradient approximation (GGA) $+\mathrm{U}$ method. It shows that the magnetism of the BLFCT is mainly originated from the transition metal ions [Fig. 3(a)], $\mathrm{Fe}^{3+}$ and $\mathrm{Co}^{3+}$ (3.1 and $2.1 \mu_{B}$ /atom, respectively), while their surrounding $\mathrm{O}$ and $\mathrm{Ti}$ atoms have a small contribution as well (less than $0.1 \mu_{B}$ /atom). The exchange processes between the adjacent corner-shared $\mathrm{FeO}_{6}$ and $\mathrm{CoO}_{6}$ tilted octahedra are dominated by the (virtual) electron transfer between the Fe/Co spin-up $\mathrm{d}_{\mathrm{xy}}$ orbitals at the Fermi level through the $\mathrm{O}$ spin-up $\mathrm{p}_{\mathrm{x}} / \mathrm{p}_{\mathrm{y}}$ orbitals [cf. Fig. 3(b)]. For characterizing the type of magnetic coupling, two different relative alignments of the magnetic moments of $\mathrm{Fe}^{3+}$ and $\mathrm{Co}^{3+}$ cations, parallel (i.e., ferromagnetic) alignment and antiparallel (i.e., antiferromagnetic) alignment, are both considered. The ferromagnetic orientation is found to have a much lower energy than the antiferromagnetic orientation, and a large energy difference reads $\mathrm{E}_{\mathrm{FM}}-\mathrm{E}_{\mathrm{AFM}}$ $=-144.1 \mathrm{meV}$. The double exchange ferromagnetism occurs in this doped system of localized spins and itinerant electrons interacting via Hund's rule coupling. The strength of the (FM) exchange interaction is around $43.5 \mathrm{meV}$ (i.e., $504.6 \mathrm{~K}$ ), which is consistent with the FM transition temperature $\mathrm{T}_{\mathrm{cl}}$ in the $\mathrm{ZFC/FC} \mathrm{measurements} \mathrm{[cf.} \mathrm{Fig.} \mathrm{2(c)].} \mathrm{The}$ parallel alignment of magnetic moments is identified within the $a-b$ plane. Together with the following neutron diffraction measurements, the in-plane orientation of magnetic moments is found to be along the [010] direction.

Figure 4 shows the neutron diffraction data collected for the BLFCT sample subjected to an external magnetic field. Intensity changes in the diffraction pattern can be observed in several peaks at large $\mathrm{d}$-spacing, while at low d-spacing, no observable intensity change

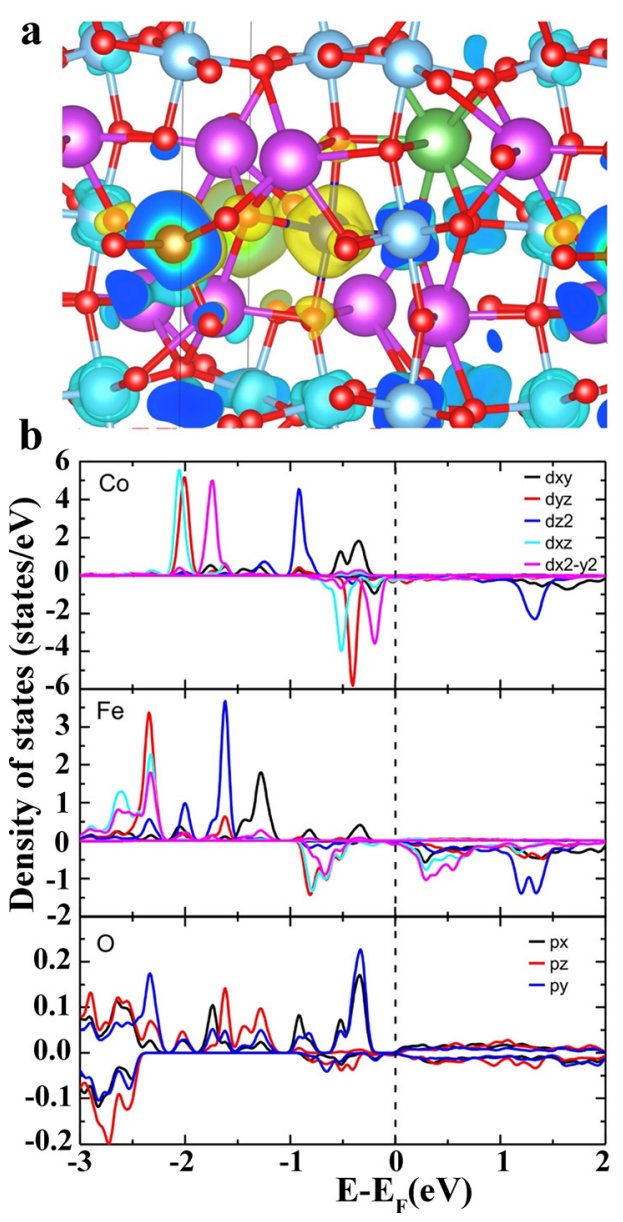

FIG. 3. (a) Spin density of the system (isosurface level $=0.005$ ): $\mathrm{O}$ (red), Bi (pink), $\mathrm{La}$ (green), Ti (light blue), Co (dark blue), and Fe (yellow) atoms. (b) A detailed inspection of the density of state in $\mathrm{Fe}^{3+}-\mathrm{O}-\mathrm{Co}^{3+}$.

is detectable. This observation is in agreement with the expected dependence from the magnetic form factor. No additional peaks could be detected, indicating that the magnetic structure is commensurate with the crystallographic structure, with the possible ferromagnetic or weak ferromagnetic character. In the inset of Fig. 4, it is shown that the intensity of the significant Aurivillius peak ( $\left.\begin{array}{lll}0 & 1 & 20\end{array}\right)$ reversibly changes under the full excursion of the applied magnetic field. The peaks' intensity increases with an increasing field of up to $2 \mathrm{~T}$. For a higher field $(2 \mathrm{~T}<H<5 \mathrm{~T})$, the peak intensity remains unchanged, which indicates that the magnetic material becomes saturated at about $2 \mathrm{~T}$, in agreement with the M-H loops in Figs. 2(d) and S3. Finally, a remanent magnetic contribution is observable when compared to the zero field measurements.

Figure 5 demonstrates the existence of magnetoelectric coupling in the BLFCT sample by the Piezoresponse Force Microscopy images of changes in the ferroelectric domain structure induced by an applied magnetic field greater than the magnetic coercive field of 399 Oe. Vertical PFM was performed on the BLFCT sample, as shown in Fig. 5(d). A partial ferroelectric domain switching observed in the local Fe- 


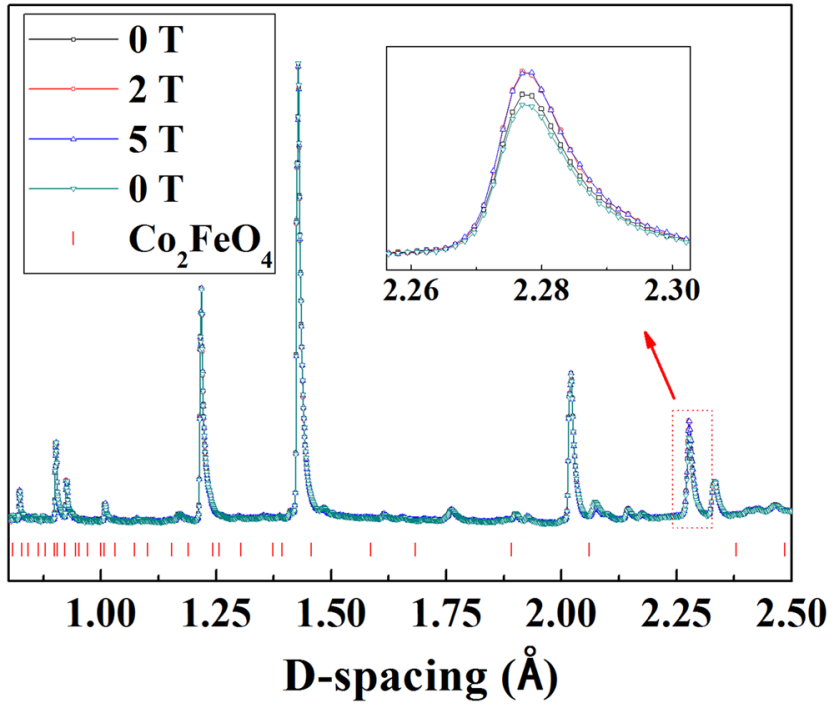

FIG. 4. The room-temperature neutron diffraction pattern obtained for the BLFCT ceramic at different magnetic fields (from 0,2 , and $5 \mathrm{~T}$ and then back to $0 \mathrm{~T}$ ).

and Co-rich nanostoichiometric regions (marked by the red circles) was obtained after applying an in-plane magnetic field of $+2000 \mathrm{Oe}$ [Fig. 5(e)]. When a magnetic field of -2000 Oe is applied in the opposite direction, part of the ferroelectric domains switches back [Fig. 5(f)]. The low content of the cubic (non-polar) secondary phase Ti doped $\mathrm{Co}_{2} \mathrm{FeO}_{4}$ and its non-ferroelectric character excludes the possibility that the described switching phenomena might be due to the secondary phase. Therefore, the magnetic-field driven changes in the ferroelectric domain structure are intrinsic, resulting from the magnetoelectric response coupling in the SPS prepared Aurivillus BLFCT ceramic. Given the fact that the ferroelectric polarization $(\boldsymbol{P})$ lies along the [100] direction and the ferromagnetic moments $(\boldsymbol{M})$ are mostly around the [010] direction, the presence of a spontaneous (ferro-

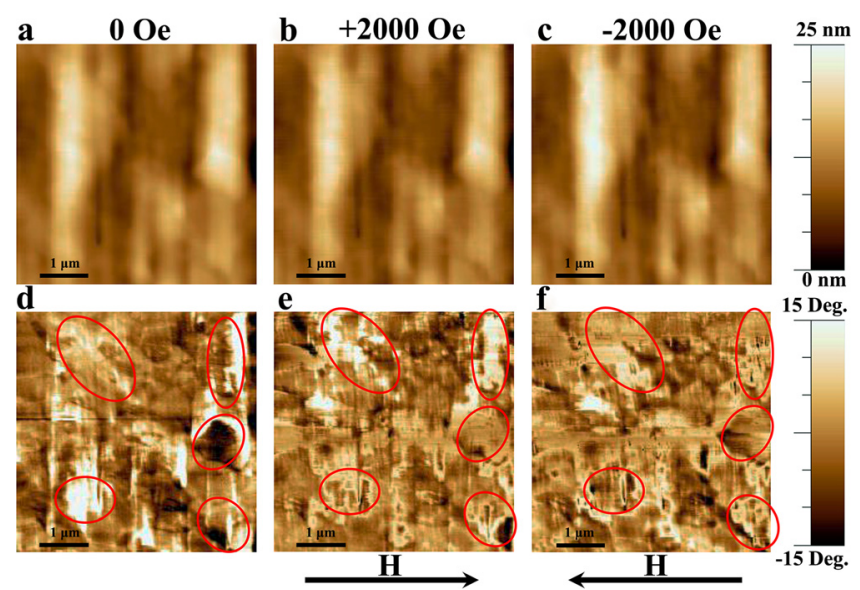

FIG. 5. Ferroelectric domain switching in BLFCT under the applied magnetic field ( $\mathrm{H}$ is parallel to the surface of the sample). (a) Topography and (d) vertical PFM phase at $0 \mathrm{Oe}$; (b) topography and (e) vertical PFM phase at $+2000 \mathrm{Oe}$; and (c) topography and (f) vertical PFM phase at -2000 Oe. )toroidal moment $\boldsymbol{T}=\boldsymbol{P} \times \boldsymbol{M}$ and a linear magnetoelectric effect is suggested to occur in BLFCT ceramics.

In conclusion, the Aurivillius phase in the BLFCT ceramic is found to be ferroelectrically and ferromagnetically active at room temperature. Ferromagnetism is suggested to originate from double exchange interactions between neighboring $\mathrm{Fe}^{3+}-\mathrm{O}-\mathrm{Fe}^{3+}$, $\mathrm{Co}^{3+}-\mathrm{O}-\mathrm{Co}^{3+}$, and $\mathrm{Fe}^{3+}-\mathrm{O}-\mathrm{Co}^{3+}$ in the main phase by the first principles calculations. The magnetic controlled ferroelectric domain switching was observed by piezoelectric force microscopy at room temperature. The Aurivillius phase ceramics, with $\mathrm{Co} / \mathrm{Fe}$ contributing to magnetization and polarization at the same time, can be considered an intrinsic room-temperature multiferroic with the ferro-toroidal phase.

See the supplementary material for more information about the details of the experiment method, the secondary phase by SEM-energy dispersive x-ray (EDX) spectroscopy, and the field dependence of magnetization of the BLFCT sample.

The authors thank Dr. Manuel, Pascal and Dr. Khalyavin, D. Dmitry for their assistance with the neutron diffraction on the WISH instrument at the ISIS Neutron Spallation facility, RAL, Didcot, Oxfordshire, UK. This study was supported by the Royal Academy of Engineering Distinguished Visiting Fellowship, the Grant Agency of the Slovak Academy of Sciences (Grant No. 2/ 0038/20), CSS (Grant No. YK2015-0602006), and NSFC (Grant Nos. 11474138 and 11834005). Additional support was funded through the European Metrology Research Programme (EMRP) Project IND54 Nanostrain. The EMRP is jointly funded by the EMRP participating countries within EURAMET and the European Union.

\section{DATA AVAILABILITY}

The data that support the findings of this study are available within the article and its supplementary material.

\section{REFERENCES}

${ }^{1}$ W. Eerenstein, N. D. Mathur, and J. F. Scott, Nature 442, 759 (2006).

${ }^{2}$ R. Ramesh and N. A. Spaldin, Nat. Mater. 6, 21 (2007).

${ }^{3}$ J. Ma, J. Hu, Z. Li, and C. W. Nan, Adv. Mater. 23, 1062 (2011).

${ }^{4}$ L. F. Henrichs, O. Cespedes, J. Bennett, J. Landers, S. Salamon, C. Heuser, T. Hansen, T. Helbig, O. Gutfleisch, D. C. Lupascu, H. Wende, W. Kleemann, and A. J. Bell, Adv. Funct. Mater. 26, 2111 (2016).

${ }^{5}$ N. A. Hill, J. Phys. Chem. B 104, 6694 (2000).

${ }^{6} \mathrm{~B}$. Aurivillius, "Mixed bismuth oxides with layer lattices: II. Structure type of $\mathrm{Bi}_{4} \mathrm{Ti}_{3} \mathrm{O}_{12}$," Arkiv. Kemi 1(58), 499-512 (1949).

${ }^{7}$ A. Srinivas, S. Suryanarayana, G. Kumar, and M. M. Kumar, J. Phys.: Condens. Mat. 11, 3335 (1999).

${ }^{8}$ N. V. Prasad and G. S. Kumar, Mater. Sci. Eng., B 108, 194 (2004).

${ }^{9}$ L. Keeney, T. Maity, M. Schmidt, A. Amann, N. Deepak, N. Petkov, S. Roy, M. E. Pemble, R. W. Whatmore, and D. Johnson, J. Am. Ceram. Soc. 96, 2339 (2013).

${ }^{10}$ Z. Li, J. Ma, Z. Gao, G. Viola, V. Koval, A. Mahajan, X. Li, C. Jia, C. Nan, and H. Yan, Dalton Trans. 45, 14049 (2016).

${ }^{11} \mathrm{~J}$. F. Scott, NPG Asia Mater. 5, e72 (2013).

${ }^{12}$ A. Y. Birenbaum and C. Ederer, Phys. Rev. B 90, 214109 (2014).

${ }^{13}$ J. B. Li, Y. P. Huang, G. H. Rao, G. Y. Liu, J. Luo, J. R. Chen, and J. K. Liang, Appl. Phys. Lett. 96, 222903 (2010).

${ }^{14}$ X. Mao, W. Wang, X. Chen, and Y. Lu, Appl. Phys. Lett. 95, 082901 (2009).

${ }^{15}$ Z. Liu, J. Yang, X. Tang, L. Yin, X. Zhu, J. Dai, and Y. Sun, Appl. Phys. Lett. 101, 122402 (2012). 
${ }^{16}$ M. Palizdar, T. P. Comyn, M. B. Ward, A. P. Brown, J. P. Harrington, S. Kulkarni, L. Keeney, S. Roy, M. Pemble, R. Whatmore, C. Quinn, S. H. Kilcoyne, and A. J. Bell, J. Appl. Phys. 112, 073919 (2012).

${ }^{17}$ J. Liu, Z. Shen, H. Yan, M. J. Reece, Y. Kan, and P. Wang, J. Appl. Phys. 102, 104107 (2007).

${ }^{18}$ M. G. Cain, Characterisation of Ferroelectric Bulk Materials and Thin Films (Springer, 2014), Vol. 2.

${ }^{19}$ Z. Li, K. Tao, J. Ma, Z. Gao, V. Koval, C. Jiang, G. Viola, H. Zhang, A. Mahajan, J. Cao, M. Cain, I. Abrahams, C. Nan, C. Ji, and H. Yan, J. Mater. Chem. C 6, 2733 (2018).
${ }^{20}$ V. Koval, I. Skorvanek, G. Viola, M. Zhang, C. Jia, and H. Yan, J. Phys. Chem. C 122, 15733 (2018).

${ }^{21}$ L. Jin, F. Li, and S. Zhang, J. Am. Ceram. Soc. 97, 1 (2014).

${ }^{22}$ H. Yan, F. Inam, G. Viola, H. Ning, H. Zhang, Q. Jiang, T. Zeng, Z. Gao, and M. J. Reece, J. Adv. Dielectr. 1, 107 (2011).

${ }^{23}$ J. Yang, L. Yin, Z. Liu, X. Zhu, W. Song, J. Dai, Z. Yang, and Y. Sun, Appl. Phys. Lett. 101, 012402 (2012).

${ }^{24}$ B. Yuan, J. Yang, J. Chen, X. Zuo, L. Yin, X. Tang, X. Zhu, J. Dai, W. Song, and Y. Sun, Appl. Phys. Lett. 104, 062413 (2014).

${ }^{25}$ I. P. Muthuselvam and R. N. Bhowmik, Solid. State. Sci. 11, 719 (2009). 